\title{
Lipocalin 2 could be the Sentinel Marker and Prognostic Factor in Subarachnoid Hemorrhage and Malignant Tumor Progression
}

\author{
Yi No Chen², Po Hui Lee ${ }^{2}$, Kuo Chuan Wang² and Te Fu Chen*1,2 \\ ${ }^{1}$ Division of Neurosurgery, Department of surgery, National Taiwan University Hospital, Taiwan \\ ${ }^{2}$ Non-invasive Cancer Therapy Research Institute, Taiwan
}

*Corresponding author: Te Fu Chen, Division of Neurosurgery, Department of surgery, National Taiwan University Hospital, Noninvasive Cancer Therapy Research Institute, Taiwan

\begin{abstract}
ARTICLE INFO
Received: March 23, 2020

Published: 慧 April 09, 2020

Citation: Yi No Chen, Po Hui Lee, Kuo Chuan Wang, Te Fu Chen. Lipocalin 2 could be the Sentinel Marker and Prognostic Factor in Subarachnoid Hemorrhage and Malignant Tumor Progression. Biomed J Sci \& Tech Res 27(1)-2020. BJSTR. MS.ID.004432.
\end{abstract}

Keywords: LCN2; SAH; AKI

\section{ABSTRACT}

Lipocalin 2 (LCN2) is a $25 \mathrm{kDa}$ protein found to be related to inflammatory modulation, cell-cell interaction, and clinical biomarker for acute renal injury. The LCN2 is highly expressed while the metastatic cancer cell invasion occurs and could be detected both in the urine or blood. Under overwhelmed inflammation, the progression of acute renal injury or cerebral ischemia could be aggravated and the serum level of LCN2 increased simultaneously. The neurological deterioration in subarachnoid hemorrhage (SAH) was closely related to neuroinflammation and inadequate microcirculation which might be LCN2 related as well. Here, the authors encourage further investigations to discover the prognostic correlation between LCN2 and SAH.

Abbreviations: LCN 2: Lipocalin 2; SAH: Subarachnoid Hemorrhage; DCI: Delayed Cerebral Ischemia; NGAL: Neutrophil Gelatinase Associated Lipocalin; AKI: Acute Kidney Injury; MMP: Matrix Metalloproteinase; BBB: Blood Brain Barrier; SNI: Spared Nerve Injury

\section{Mini Review}

Cerebral vasospasm, along with succeeding delayed cerebral ischemia (DCI), are notoriously severe complication subsequent to subarachnoid hemorrhage (SAH). The overwhelmed neuroinflammation plays a crucial role behind while causing a direct brain injury [1]. As injured brain tissue fail to function normally due to deteriorated microcirculation, "vasoconstriction" rather than vasodilation take place with sequential ischemia in a vicious cycle. How to detect the process earlier and give a proper clinical treatment response were always challenging the clinical physician. However, current monitoring methods such as beside transcranial duplex study, computed tomography angiography, or traditional cerebral angiography may require skilled examiner or the exposure to radiation [2]. A reliable biomarker is warranted for monitoring the severity or the progression in SAH. Lipocalin 2 may be a candidate for further study in SAH model. Lipocalin 2 (LCN2), or neutrophil gelatinase associated lipocalin (NGAL), is a $25 \mathrm{kDa}$ protein sharing general structure of lipocalin family with hydrophobic core, which facilitates its binding with lipophilic substances [3].

It was known for its association with inflammatory response in heart [4,5], bowel [6] or even CNS [7]. First purified by Kjeldsen et al. [7] as a 25-kDa protein from neutrophil granules, it was later found by Bundgaard et al. [8] of its large amounts of expression in myeloid cells [8]. Later on, LCN2 plays a crucial role in immunity was hypothesized after the disclosure of the involvement in intercellular iron delivery mechanism [9]. Goetz and his coworkers pointed out that the specificity, along with affinity of LCN2 for bacterial catecholate-type ferric siderophores, which takes part in antibacterial iron-depletion strategy of immune system and explains the phenomena of high serum concentration during inflammation [10]. The hypothesis was proved later in vitro with LCN2 deficient mice with isolated neutrophils showing less bacteriostatic activity [11-13], and in vivo with higher bacterial numbers found in bladders 
of LCN2-deficient mice than wild-type mice [14]. This contributes to the prognostic value of acute kidney injury (AKI), which is highly excreted as tubular injury occurs $[15,16]$. A meta-analysis of data from 19 studies elucidated that urine NGAL levels were found to be diagnostic for AKI [17].

LCN2 covalently binds matrix metalloproteinase-9 (MMP), a gelatinase, or proteolytic enzymes secreted by neutrophils. As a member of superfamily of proteolytic enzymes, MMP-9 is related to disruption of blood brain barrier (BBB) [18]. It was reported that NGAL prevents MMP-9 from degradation [19], and in certain degree contributes to the tumor progression. Independently, LCN2 increases the invasiveness of certain cancer cells, while inhibition of LCN2 expression decreases their invasiveness [20]. In a study designed to examine the difference of LCN2 and MMP-9 expression in the tumor tissues from patients with lung adenocarcinoma, LCN2 expression turned out to be an independent prognostic factor, while expression of MMP-9 failed to indicate overall survival rate difference [21]. More studies showed NGAL/MMP-9 complex seemingly elevates in urine of cancer patients, which may imply the participation of this $125 \mathrm{kDa}$ complex in tumor progression [19,2224]. However, the physiological and pathological process behind NGAL/MMP-9 complex is still not fully known currently.

Two receptors of LCN2 have been identified, the solute carrier family 22 member 17 (SLC22A17 or 24p3R) and megalin, as the former is located in hippocampus [25], choroid plexus [26], whilst the latter is positioned throughout the brain, expressed by miscellaneous types of cells: neurons, endothelial cells and astrocytes. 24p3R is a cell surface receptor for LCN2 expressed in various organs, which modulates the cellular uptake of LCN2 and diverse physiological processes. Using the spared nerve injury (SNI) model [27], Sangmin et al. found the expression of 24p3R in microglia as well as neurons, while high levels of 24p3R was present in the normal spinal cord. Khizr I et al. suggested the participation of LCN2 in regulating inflammation in the injured spinal cord and 24p3R-expressing spinal neurons is probably sensitive to LCN2. These findings showed that $24 \mathrm{p} 3 \mathrm{R}$, largely expressed in neurons and to some degree in microglia, may modulate the actions of LCN2 under the condition of neuropathic pain. By acting on its receptor, $24 \mathrm{p} 3 \mathrm{R}$, which is spread throughout the spinal cord, LCN2 is virtually involved in the development of pain hypersensitivity [27].

The idea of taking LCN2 as "target cell" gets even stronger when there's a better recovery outcome for spinal injury due to lack of LCN2 [28] and modulation of LCN2 might attenuate Alzheimer's disease [29]. However, there is report that showed seemingly controversial result that LCN2 deficiency had no effect on aggravation of systemic disease [30], supporting the notion of regarding LCN2 as a biomarker, which doesn't have impact on the disease itself while upregulated as disease deteriorates. Hochmeister et al. [31] supported the evidence for regarding LCN2 as biomarker and elucidated that plasma LCN2 level predicts clinical outcome in ischemic stroke model [31]. SAH is a hemorrhagic intracerebral ischemic process and brings catastrophic change to the microenvironment in the brain. The severity of blood brain barrier (BBB) breakdown had high correlation to neuroinflammation and poorer outcome [32], while the hyperpermeability of BBB increase the neutrophils migration toward the brain parenchyma would increase neuroinflammation. LCN2 might be the ideal serum biomarker of SAH disease progression [33] while more clinical studies should be encouraged.

\section{References}

1. Miller BA, Nefize T, Monica C, Gustavo P (2014) Inflammation, vasospasm, and brain injury after subarachnoid hemorrhage. Biomed Res Int pp. 384342.

2. Mohamed S, A Yazan (2017) Management of cerebral vasospasm following aneurysmal subarachnoid hemorrhage. The Southwest Respiratory and Critical Care Chronicles 5(20).

3. Kjeldsen L, Bainton DF, Sengeløv H, Borregaard N (1994) Identification of neutrophil gelatinase-associated lipocalin as a novel matrix protein of specific granules in human neutrophils. Blood 83(3): 799-807.

4. Aigner F, Maier HT, Schwelberger HG, Wallnöfer EA, Amberger A, et al. (2007) Lipocalin-2 regulates the inflammatory response during ischemia and reperfusion of the transplanted heart. Am J Transplant 7(4): 779-788.

5. Marques FZ, Prestes PR, Byars SG, Ritchie SC, Würtz P, et al. (2017) Experimental and Human Evidence for Lipocalin-2 (Neutrophil Gelatinase-Associated Lipocalin [NGAL]) in the Development of Cardiac Hypertrophy and heart failure. J Am Heart Assoc 6(6).

6. Moschen AR, Adolph TE, Gerner RR, Wieser V, Tilg H, et al. (2017) Lipocalin-2: A Master Mediator of Intestinal and Metabolic Inflammation. Trends Endocrinol Metab 28(5): 388-397.

7. Meyerhoff N, Rohn K, Carlson R, Tipold A (2019) Measurement of Neutrophil Gelatinase-Associated Lipocalin Concentration in Canine Cerebrospinal Fluid and Serum and Its Involvement in Neuroinflammation. Front Vet Sci 6: 315.

8. Bundgaard JR, Sengel $\varnothing v$ H, Borregaard N, Kjeldsen L (1994) Molecular cloning and expression of a cDNA encoding NGAL: a lipocalin expressed in human neutrophils. Biochem Biophys Res Commun 202(3): 14681475 .

9. Goetz DH, Holmes MA, Borregaard N, Bluhm ME, Raymond KN, et al. (2002) The neutrophil lipocalin NGAL is a bacteriostatic agent that interferes with siderophore-mediated iron acquisition. Mol Cell 10(5): 1033-1043.

10. Xu S, P Venge (2000) Lipocalins as biochemical markers of disease. Biochim Biophys Acta 1482(1-2): 298-307.

11. Berger T, Togawa A, Duncan GS, Elia AJ, You Ten A, et al. (2006) Lipocalin 2-deficient mice exhibit increased sensitivity to Escherichia coli infection but not to ischemia-reperfusion injury. Proc Natl Acad Sci U S A 103(6): 1834-1839.

12. Nairz M, Schroll A, Haschka D, Dichtl S, Sonnweber T, et al. (2015) Lipocalin-2 ensures host defense against Salmonella Typhimurium by controlling macrophage iron homeostasis and immune response. Eur J Immunol 45(11): 3073-3086.

13. Wang Q Li S, Tang X, Liang L, Wang F, et al. (2019) Lipocalin 2 Protects Against Escherichia coli Infection by Modulating Neutrophil and Macrophage Function. Front Immunol 10: 2594.

14. Steigedal M, Marstad A, Haug M, Damås JK, Strong RK, et al. (2014) Lipocalin 2 imparts selective pressure on bacterial growth in the 
bladder and is elevated in women with urinary tract infection. J Immunol 193(12): 6081-6089.

15. Devarajan P (2008) Neutrophil gelatinase-associated lipocalin--an emerging troponin for kidney injury. Nephrol Dial Transplant 23(12): 3737-3743.

16. Treeprasertsuk S, Wongkarnjana A, Jaruvongvanich V, Sallapant S, Tiranathanagul K, et al. (2015) Urine neutrophil gelatinase-associated lipocalin: a diagnostic and prognostic marker for acute kidney injury (AKI) in hospitalized cirrhotic patients with AKI-prone conditions. BMC Gastroenterol 15: 140 .

17. Haase M, Bellomo R, Devarajan P, Schlattmann P, Haase Fielitz A, et al. (2009) Accuracy of neutrophil gelatinase-associated lipocalin (NGAL) in diagnosis and prognosis in acute kidney injury: a systematic review and meta-analysis. Am J Kidney Dis 54(6): 1012-1024.

18. Feng S (2011) Matrix metalloproteinase-2 and -9 secreted by leukemic cells increase the permeability of blood-brain barrier by disrupting tight junction proteins. PLoS One 6(8): e20599.

19. Yan L, Borregaard N, Kjeldsen L, Moses MA (2001) The high molecular weight urinary matrix metalloproteinase (MMP) activity is a complex of gelatinase B/MMP-9 and neutrophil gelatinase-associated lipocalin (NGAL). Modulation of MMP-9 activity by NGAL. J Biol Chem 276(40): 37258-37265.

20. Du ZP, Wu BL, Xie YM, Zhang YL, Liao LD, et al. (2015) Lipocalin 2 promotes the migration and invasion of esophageal squamous cell carcinoma cells through a novel positive feedback loop. Biochim Biophys Acta 1853(10 Pt A): 2240-2250.

21. Ruiz Morales JM, Dorantes Heredia R, Arrieta O, Chávez Tapia NC, Motola Kuba D (2015) Neutrophil gelatinase-associated lipocalin (NGAL) and matrix metalloproteinase-9 (MMP-9) prognostic value in lung adenocarcinoma. Tumour Biol 36(5): 3601-3610.

22. Kjeldsen L, Johnsen AH, Sengel $\varnothing v H$, Borregaard N (1993) Isolation and primary structure of NGAL, a novel protein associated with human neutrophil gelatinase. J Biol Chem 268(14): 10425-10432.

23. Liu MF, Hu YY, Jin T, Xu K, Wang SH, et al. (2015) Matrix Metalloproteinase-9/Neutrophil Gelatinase-Associated Lipocalin
Complex Activity in Human Glioma Samples Predicts Tumor Presence and Clinical Prognosis. Dis Markers 2015: 138974.

24. Shimura T, Dagher A, Sachdev M, Ebi M, Yamada T, et al. (2015) Urinary ADAM12 and MMP-9/NGAL complex detect the presence of gastric cancer. Cancer Prev Res (Phila) 8(3): 240-248.

25. Chia WJ, Tan FCK, Ong WY, Dawe GS (2015) Expression and localisation of brain-type organic cation transporter (BOCT/24p3R/LCN2R) in the normal rat hippocampus and after kainate-induced excitotoxicity. Neurochem Int 87: 43-59.

26. Nagle MA, Wu W, Eraly SA, Nigam SK (2013) Organic anion transport pathways in antiviral handling in choroid plexus in Oat1 (Slc22a6) and Oat3 (Slc22a8) deficient tissue. Neurosci Lett 534: 133-138.

27. Jeon S, Jha MK, Ock J, Seo J, Jin M, et al. (2013) Role of lipocalin-2chemokine axis in the development of neuropathic pain following peripheral nerve injury. J Biol Chem 288(33): 24116-24127.

28. Rathore KI, Berard JL, Redensek A, Chierzi S, Lopez Vales R, et al. (2011) Lipocalin 2 plays an immunomodulatory role and has detrimental effects after spinal cord injury. J Neurosci 31(38): 13412-13419.

29. Song J, OY Kim (2018) Perspectives in Lipocalin-2: Emerging Biomarker for Medical Diagnosis and Prognosis for Alzheimer's Disease. Clin Nutr Res 7(1): 1-10.

30. Mike EV, Makinde HM, Gulinello M, Vanarsa K, Herlitz L, et al (2019) Lipocalin-2 is a pathogenic determinant and biomarker of neuropsychiatric lupus. J Autoimmun 96: 59-73.

31. Hochmeister S, Engel O, Adzemovic MZ, Pekar T, Kendlbacher P, et al. (2016) Lipocalin-2 as an Infection-Related Biomarker to Predict Clinical Outcome in Ischemic Stroke. PLoS One 11(5): e0154797.

32. Abbott NJ (2000) Inflammatory mediators and modulation of bloodbrain barrier permeability. Cell Mol Neurobiol 20(2): 131-147.

33. Egashira Y, Hua Y, Keep RF, Iwama T, Xi G (2016) Lipocalin 2 and Blood-Brain Barrier Disruption in White Matter after Experimental Subarachnoid Hemorrhage. Acta Neurochir Suppl 121: 131-134.

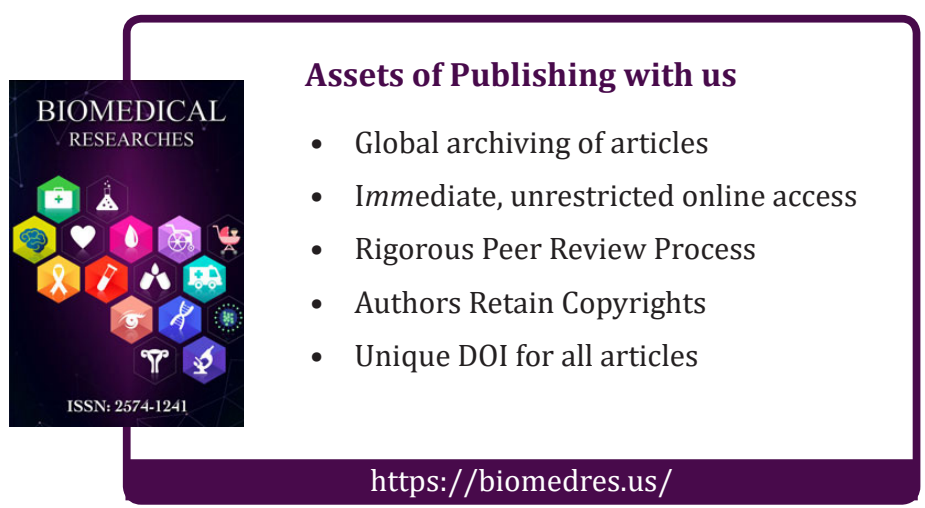

